SUPPORTING INFORMATION.

\title{
Metabolomics investigation of voriconazole-induced hepatotoxicity in
} mice

Shin-Lun Wu ${ }^{1}$, Ting-Yu Wei ${ }^{1}$, Shu-Wen Lin ${ }^{1}$, Kang-Yi Su ${ }^{* 2,3,4,5}$, Ching-Hua Kuo ${ }^{* 1,5,6}$

${ }^{1}$ School of Pharmacy, College of Medicine, National Taiwan University, Taiwan;

${ }^{2}$ Department of Clinical Laboratory Sciences and Medical Biotechnology, College of Medicine, National Taiwan University, Taiwan;

${ }^{3}$ Department of Laboratory Medicine, National Taiwan University Hospital, Taiwan;

${ }^{4}$ Genome and Systems Biology Degree Program, National Taiwan University and Academia Sinica, Taiwan.

${ }^{5}$ Centers of Genomic and Precision Medicine, National Taiwan University, Taiwan.

${ }^{6}$ Department of Pharmacy, National Taiwan University Hospital, Taiwan.

*Corresponding Authors

Kang-Yi Su

Address: Department of Clinical Laboratory Science and Medical Biotechnology, National Taiwan University. No.1, Sec.1, Jen-I Road, Taipei 10055, Taiwan. R.O.C.

Tel: +886.2.23123456 ext. 66910

Fax: +886.2 .23711574$

E-mail: suky@ntu.edu.tw

Ching-Hua Kuo

Address: School of Pharmacy, College of Medicine, National Taiwan University, Rm. 418, 4F., No.33, Linsen S. Rd., Chongsheng Dist., Taipei City 100, Taiwan (R.O.C.)

Tel: +886.2 .33668766$

Fax: +886.2 .23919098$

E-mail: kuoch@ntu.edu.tw 


\section{This SI contains the following:}

Figure S1 Correlations between levels of acylcarnitines and GOT/AST. The levels of acylcarnitines are highly correlated to the level of GOT/AST. Mice with VCZ treatments (red circle) exhibit relative higher GOT/AST compared with control ones.

.page $\mathrm{S}-3$

Figure S2 Correlations between levels of acylcarnitines and GPT/ALT. The levels of acylcarnitines are highly correlated to the level of GPT/ALT. Mice with VCZ treatments (red circle) exhibit relative higher GPT/ALT compared with control ones.

page $\mathrm{S}-4$

Table S1 Mass transitions and retention times of the target metabolites. ...page S-5

Table S2 Mass parameters, transitions and retention times for profiling acylcarnitines. page S-10

Table S3 Peak area table of targeted metabolomics analysis in liver samples. Only the targeted metabolites with RSD no more than $20 \%$ in QC samples are shown.

page $\mathrm{S}-12$

Table S4 Peak area table of targeted metabolomics analysis in plasma samples. Only the targeted metabolites with RSD no more than $20 \%$ in QC samples are shown.

.page $\mathrm{S}-15$

Table S5 Metabolites with fold change less than 0.8 or over 1.2 in liver and plasma samples. 

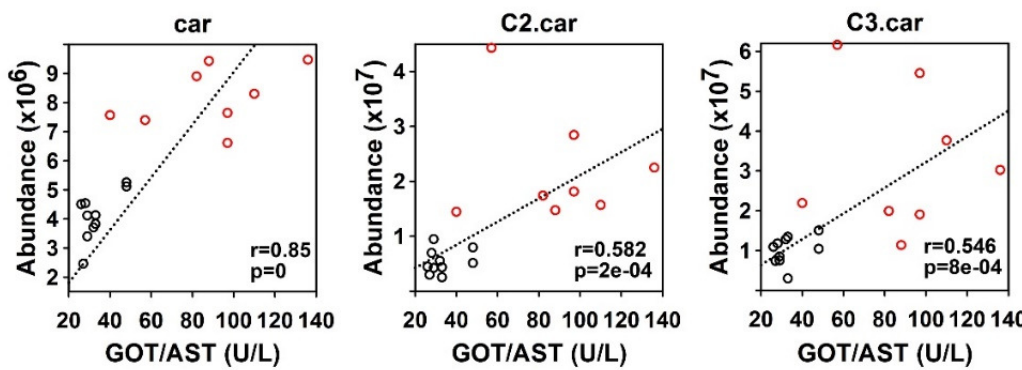

$204060 \quad 80100120140$ GOT/AST (U/L)

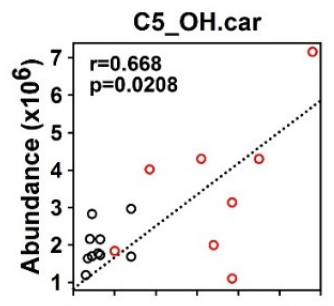

20406080100120140 GOT/AST (U/L)
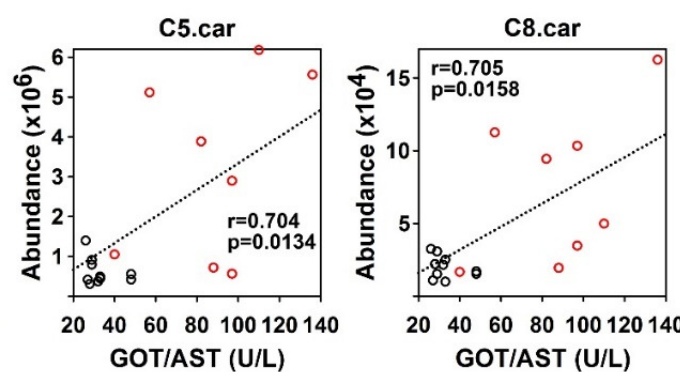

$2040 \quad 6080100120140$ GOT/AST (U/L)
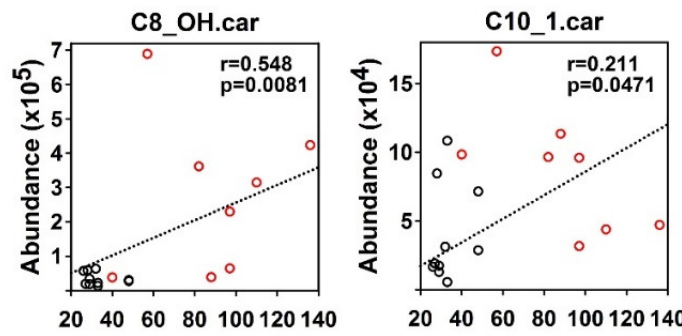

$20 \quad 40 \quad 60 \quad 80100120140$ GOT/AST (U/L)
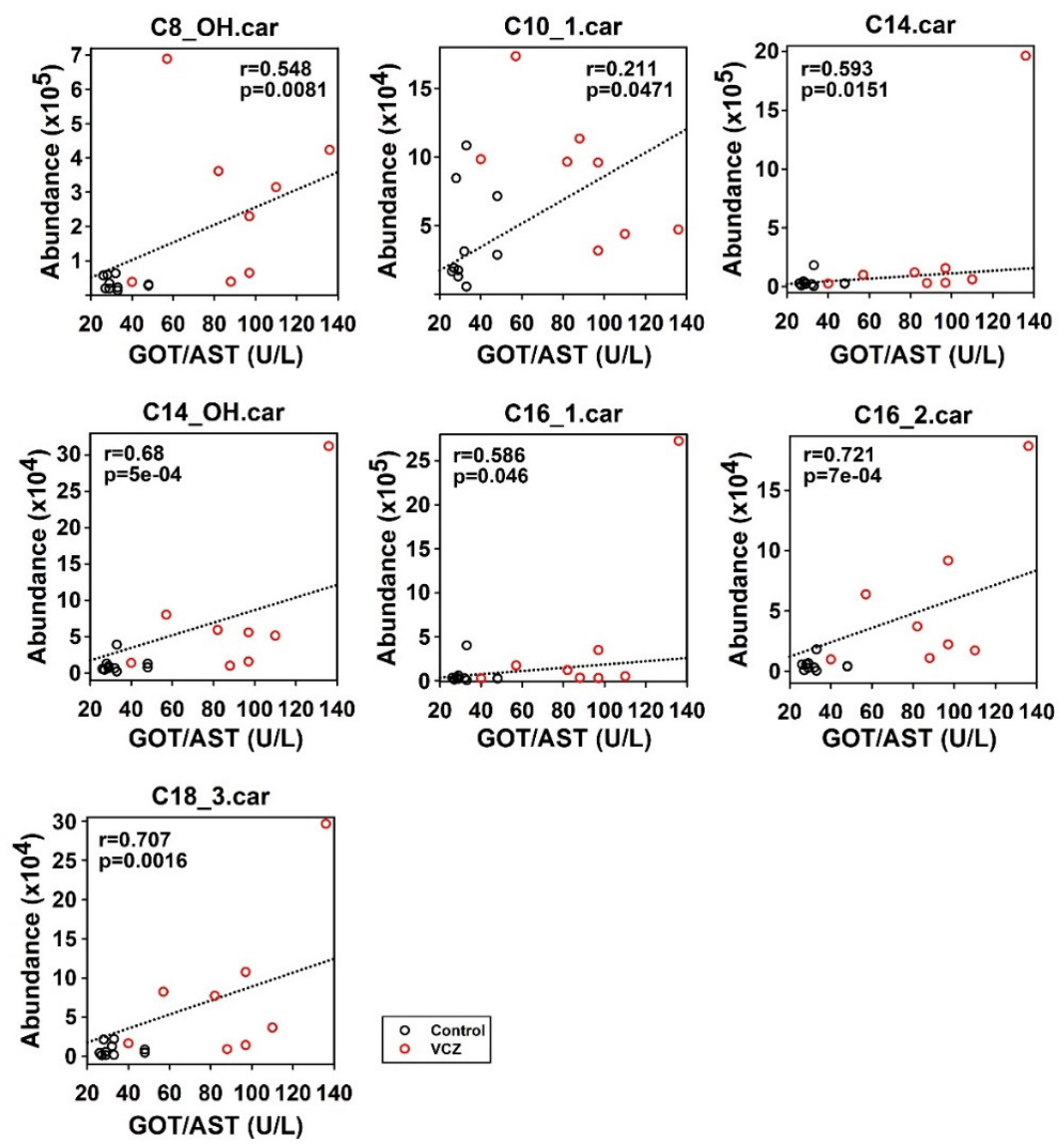
GOT/AST (U/L)

Figure S1 Correlations between levels of acylcarnitines and GOT/AST. The levels of acylcarnitines are highly correlated to the level of GOT/AST. Mice with VCZ treatments (red circle) exhibit relative higher GOT/AST compared with control ones. 

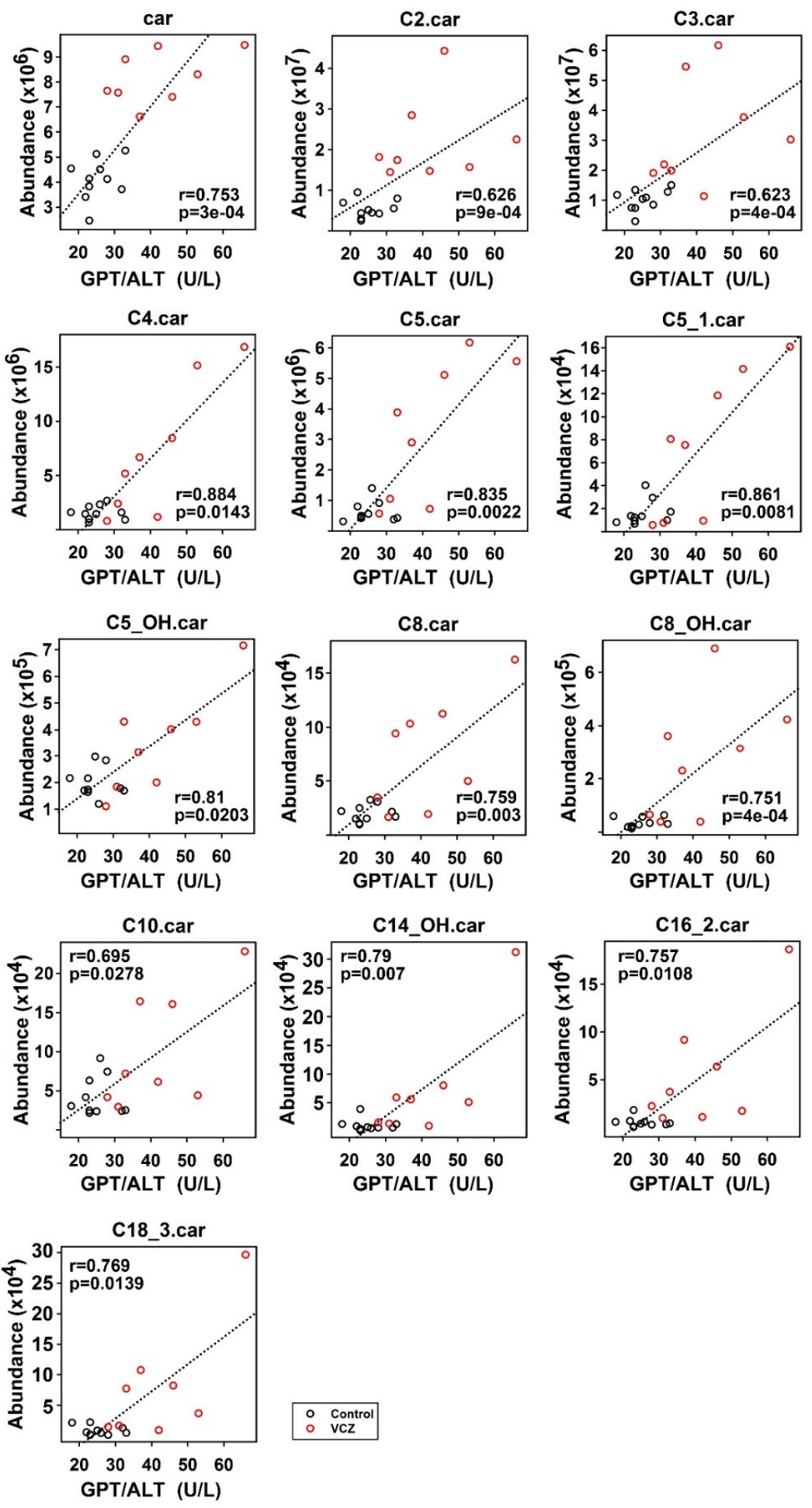

Figure S2 Correlations between levels of acylcarnitines and GPT/ALT. The levels of acylcarnitines are highly correlated to the level of GPT/ALT. Mice with VCZ treatments (red circle) exhibit relative higher GPT/ALT compared with control ones. 
Table S1 Mass transitions and retention times of the target metabolites.

\begin{tabular}{|c|c|c|c|c|}
\hline Compound Name & Ion Function & Transition (POS) & Transition (NEG) & $\mathrm{RT}^{*}(\min )$ \\
\hline \multicolumn{5}{|l|}{ Nucleosides } \\
\hline \multirow{2}{*}{ Cytidine } & Qualifier & $244.1->95.1$ & & \multirow{2}{*}{1.42} \\
\hline & Quantifier & $244.1->112$ & & \\
\hline \multirow{2}{*}{ Inosine } & Qualifier & $269.1->119.1$ & & \multirow{2}{*}{1.13} \\
\hline & Quantifier & $269.1->137$ & & \\
\hline \multirow{2}{*}{ Uridine } & Qualifier & $245.1->70.1$ & & \multirow{2}{*}{1.42} \\
\hline & Quantifier & $245.1->113$ & & \\
\hline \multicolumn{5}{|l|}{ Amino acids } \\
\hline \multirow{2}{*}{ 1-Methylhistidine } & Qualifier & $170.1->83.1$ & & \multirow{2}{*}{5.24} \\
\hline & Quantifier & $170.1->124.1$ & & \\
\hline \multirow{2}{*}{ 3-Methylhistidine } & Qualifier & $170.1->95.1$ & & \multirow{2}{*}{5.50} \\
\hline & Quantifier & $170.1->109$ & & \\
\hline \multirow{2}{*}{ 5-Hydroxylysine } & Qualifier & $163.1->55.1$ & $161.1->143.1$ & \multirow{2}{*}{5.45} \\
\hline & Quantifier & $163.1->128$ & $161.1->115$ & \\
\hline \multirow{2}{*}{ Alanine } & Qualifier & $90.1->42.2$ & & \multirow{2}{*}{3.48} \\
\hline & Quantifier & $90.1->44.2$ & & \\
\hline \multirow{2}{*}{ Arginine } & Qualifier & $175.1->60.1$ & $173.1->156.1$ & \multirow{2}{*}{5.17} \\
\hline & Quantifier & $175.1->70.1$ & $173.1->131$ & \\
\hline \multirow{2}{*}{ Asparagine } & Qualifier & $133.1->87$ & & \multirow{2}{*}{3.70} \\
\hline & Quantifier & $133.1->74$ & & \\
\hline \multirow{2}{*}{ Aspartate } & Qualifier & $134->74$ & & \multirow{2}{*}{3.16} \\
\hline & Quantifier & $134->88$ & & \\
\hline \multirow{2}{*}{$\beta$-alanine } & Qualifier & $90.1->72.1$ & & \multirow{2}{*}{3.18} \\
\hline & Quantifier & $90.1->30.2$ & & \\
\hline \multirow{2}{*}{ Creatinine } & Qualifier & $114.1->86.1$ & & \multirow{2}{*}{1.77} \\
\hline & Quantifier & $114.1->44.1$ & & \\
\hline \multirow{2}{*}{ Cystine } & Qualifier & $241->151.9$ & & \multirow{2}{*}{5.44} \\
\hline & Quantifier & $241->74$ & & \\
\hline
\end{tabular}


Table S1 Mass transitions and retention times of the target metabolites. (continued)

\begin{tabular}{|c|c|c|c|c|}
\hline Compound Name & Ion Function & Transition (POS) & Transition (NEG) & $\mathrm{RT}^{*}(\min )$ \\
\hline Dimethyl & Qualifier & $104.1->42.1$ & & \multirow{2}{*}{3.78} \\
\hline Glycine & Quantifier & $104.1->58.1$ & & \\
\hline \multirow{2}{*}{ Glutamate } & Qualifier & $148.1->130$ & $146->127.9$ & \multirow{2}{*}{3.18} \\
\hline & Quantifier & $148.1->84.1$ & $146->102$ & \\
\hline \multirow{2}{*}{ Glutamine } & Qualifier & & $145.1->42.1$ & \multirow{2}{*}{3.96} \\
\hline & Quantifier & & $145.1->126.9$ & \\
\hline \multirow{2}{*}{ Glycine } & Qualifier & $76->48.2$ & & \multirow{2}{*}{3.64} \\
\hline & Quantifier & $76->30.2$ & & \\
\hline \multirow{2}{*}{ Hippurate } & Qualifier & $180.1->77.1$ & & \multirow{2}{*}{0.71} \\
\hline & Quantifier & $180.1->105$ & & \\
\hline \multirow{2}{*}{ Histidine } & Qualifier & $156.1->56.1$ & & \multirow{2}{*}{5.04} \\
\hline & Quantifier & $156.1->110$ & & \\
\hline \multirow{2}{*}{ Isoleucine } & Qualifier & $132.1->41.2$ & & \multirow{2}{*}{2.74} \\
\hline & Quantifier & $132.1->86.1$ & & \\
\hline \multirow{2}{*}{ Lysine } & Qualifier & $147.1->130$ & & \multirow{2}{*}{5.37} \\
\hline & Quantifier & $147.1->84.1$ & & \\
\hline \multirow{2}{*}{ Phenylalanine } & Qualifier & $166.1->103$ & $164.1->103$ & \multirow{2}{*}{2.62} \\
\hline & Quantifier & $166.1->120$ & $164.1->146.9$ & \\
\hline \multirow{2}{*}{ Serine } & Qualifier & $106.1->42.1$ & & \multirow{2}{*}{3.44} \\
\hline & Quantifier & $106.1->60.1$ & & \\
\hline \multirow{2}{*}{ Threonine } & Qualifier & $120.1->74.1$ & & \multirow{2}{*}{3.24} \\
\hline & Quantifier & $120.1->56.1$ & & \\
\hline \multirow{2}{*}{ Tryptophan } & Qualifier & $205.1->146$ & $203.1->159.1$ & \multirow{2}{*}{2.51} \\
\hline & Quantifier & $205.1->188$ & $203.1->116$ & \\
\hline \multirow{2}{*}{ Tyrosine } & Qualifier & $182.1->165$ & & \multirow{2}{*}{2.77} \\
\hline & Quantifier & $182.1->136$ & & \\
\hline \multirow{2}{*}{ Urocanate } & Qualifier & $139->93$ & $137->40.1$ & \multirow{2}{*}{0.95} \\
\hline & Quantifier & $139->121$ & $137->93$ & \\
\hline
\end{tabular}


Table S1 Mass transitions and retention times of the target metabolites. (continued)

\begin{tabular}{|c|c|c|c|c|}
\hline Compound Name & Ion Function & Transition (POS) & Transition (NEG) & $\mathrm{RT}^{*}$ (min.) \\
\hline \multirow{2}{*}{ Valine } & Qualifier & $118.1->55.1$ & & \multirow{2}{*}{3.04} \\
\hline & Quantifier & $118.1->72.1$ & & \\
\hline \multirow{2}{*}{$\begin{array}{l}\text { Glutathione } \\
\text { (Reduced) }\end{array}$} & Qualifier & $308.1->76.1$ & & \multirow{2}{*}{5.95} \\
\hline & Quantifier & $308.1->179$ & & \\
\hline \multirow{2}{*}{ Pyroglutamate } & Qualifier & $130->56.2$ & & \multirow{2}{*}{1.17} \\
\hline & Quantifier & $130->84.1$ & & \\
\hline \multirow{2}{*}{ Methionine } & Qualifier & $150.1->133$ & & \multirow{2}{*}{2.79} \\
\hline & Quantifier & $150.1->104$ & $148->47$ & \\
\hline \multicolumn{5}{|c|}{ Urea cycle metabolite } \\
\hline \multirow{2}{*}{ Urea } & Qualifier & $61->43.1$ & & \multirow{2}{*}{0.98} \\
\hline & Quantifier & $61->44.1$ & & \\
\hline \multirow{2}{*}{ Citrulline } & Qualifier & $176.1->159$ & & \multirow{2}{*}{4.52} \\
\hline & Quantifier & $176.1->70.1$ & & \\
\hline \multirow{2}{*}{ Ornithine } & Qualifier & $133.1->116.1$ & & \multirow{2}{*}{5.30} \\
\hline & Quantifier & $133.1->70.1$ & & \\
\hline \multicolumn{5}{|l|}{ Bile acids } \\
\hline \multirow{2}{*}{ Chenodeoxycholate } & Qualifier & $393.3->357.2$ & $391.3->392.4$ & \multirow{2}{*}{0.58} \\
\hline & Quantifier & $393.3->76.9$ & $391.3->373.3$ & \\
\hline \multirow{2}{*}{ Cholate } & Qualifier & $355.3->115$ & $407.3->69.1$ & \multirow{2}{*}{0.81} \\
\hline & Quantifier & $355.3->91.1$ & $407.3->287.2$ & \\
\hline \multirow{2}{*}{ Glycocholate } & Qualifier & $466.3->91.1$ & $464.3->400.1$ & \multirow{2}{*}{1.15} \\
\hline & Quantifier & $448.4->430.3$ & $464.3->74$ & \\
\hline \multirow{2}{*}{ Taurine } & Qualifier & $126->108$ & $123.9->64.1$ & \multirow{2}{*}{1.10} \\
\hline & Quantifier & $126->44.2$ & $123.9->79.9$ & \\
\hline \multirow{2}{*}{ Ursodeoxycholate } & Qualifier & $393.3->150$ & & \multirow{2}{*}{0.58} \\
\hline & Quantifier & $393.3->148.9$ & & \\
\hline \multirow{2}{*}{ Deoxycholate } & Qualifier & & $391.3->343.3$ & \multirow{2}{*}{0.63} \\
\hline & Quantifier & & $391.3->345.3$ & \\
\hline
\end{tabular}


Table S1 Mass transitions and retention times of the target metabolites. (continued)

\begin{tabular}{|c|c|c|c|c|}
\hline Compound Name & Ion Function & Transition (POS) & Transition (NEG) & $\mathrm{RT}^{*}(\min )$ \\
\hline \multirow{2}{*}{ Lithocholate } & Qualifier & & $375.3->374$ & \multirow{2}{*}{0.59} \\
\hline & Quantifier & & $375.3->376.4$ & \\
\hline Bilirubin & Quantifier & $585.3->299.1$ & & 0.59 \\
\hline \multicolumn{5}{|l|}{ Energy metabolites } \\
\hline \multirow{2}{*}{ Acetylcarnitine } & Qualifier & $204.1->60.2$ & & \multirow{2}{*}{1.10} \\
\hline & Quantifier & $204.1->85.1$ & & \\
\hline \multirow{2}{*}{$\alpha$-ketoglutarate } & Qualifier & & $145->57$ & \multirow{2}{*}{1.08} \\
\hline & Quantifier & & $145->101$ & \\
\hline \multirow{2}{*}{ Pyruvate } & Qualifier & & & \multirow{2}{*}{0.54} \\
\hline & Quantifier & & $87.0->43.1$ & \\
\hline \multirow{2}{*}{$\begin{array}{c}\text { Uridine Diphosphate } \\
\text { (UDP) }\end{array}$} & Qualifier & $405->97$ & & \multirow{2}{*}{0.59} \\
\hline & Quantifier & $405->41.3$ & & \\
\hline \multirow{2}{*}{ Fumarate } & Qualifier & & $115->114.5$ & \multirow{2}{*}{0.70} \\
\hline & Quantifier & & $115->70.9$ & \\
\hline \multirow{2}{*}{ Succinate } & Qualifier & & $99.2->54.9$ & \multirow{2}{*}{0.65} \\
\hline & Quantifier & & $117.9->72.9$ & \\
\hline \multicolumn{5}{|l|}{ Others } \\
\hline $17 \alpha-$ & Qualifier & $333.2->65.1$ & & \multirow{2}{*}{0.64} \\
\hline Hydroxypregnenolone & Quantifier & $333.2->333$ & & \\
\hline$\beta-\mathrm{N}-$ & Qualifier & $222.1->204$ & & \multirow{2}{*}{1.22} \\
\hline Acetylglucosamine & Quantifier & $222.1->138$ & & \\
\hline \multirow{2}{*}{ Acetoacetate } & Qualifier & $103->29.2$ & & \multirow{2}{*}{5.05} \\
\hline & Quantifier & $103->30.2$ & & \\
\hline \multirow{2}{*}{ Betaine } & Qualifier & $118.1->58.1$ & & \multirow{2}{*}{3.05} \\
\hline & Quantifier & $118.1->42.1$ & & \\
\hline \multirow{2}{*}{ Cadaverine } & Qualifier & $103.2->41.2$ & & \multirow{2}{*}{5.04} \\
\hline & Quantifier & $103.1->86.1$ & & \\
\hline \multirow{2}{*}{ Creatine } & Qualifier & $132.1->90.1$ & & \multirow{2}{*}{3.77} \\
\hline & Quantifier & $132.1->44.2$ & & \\
\hline
\end{tabular}


Table S1 Mass transitions and retention times of the target metabolites. (continued)

\begin{tabular}{ccccc}
\hline Compound Name & Ion Function & Transition (POS) & Transition (NEG) & RT $^{*}$ (min) \\
\hline \multirow{2}{*}{ Corticosterone } & Qualifier & $347.2->121$ & & 0.64 \\
& Quantifier & $347.2->329.1$ & & \\
GABA & Qualifier & $104.1->45.1$ & & 2.67 \\
& Quantifier & $104.1->87.1$ & & \\
TMAO & Qualifier & $76.1->59.1$ & & 2.70 \\
& Quantifier & $76.1->58.1$ & & 3.18 \\
Ureidopropionate & Qualifier & & $131->41.8$ & \\
& Quantifier & & $131->88$ & 0.64 \\
& Qualifier & & $143->41.1$ & \\
\hline
\end{tabular}


Table S2 Mass parameters, transitions and retention times for profiling acylcarnitines.

\begin{tabular}{|c|c|c|c|c|c|}
\hline $\begin{array}{l}\text { Compound } \\
\text { Name }\end{array}$ & $\begin{array}{l}\text { Precursor Ion } \\
(\mathrm{m} / \mathrm{z})\end{array}$ & $\begin{array}{l}\text { Product Ion } \\
(\mathrm{m} / \mathrm{z})\end{array}$ & $\begin{array}{l}\text { Fragmentor } \\
\text { (V) }\end{array}$ & $\begin{array}{l}\text { Collision Energy } \\
\text { (V) }\end{array}$ & $\begin{array}{l}\text { Rt } \\
(\min )\end{array}$ \\
\hline car & 162 & 85 & 100 & 20 & 0.7 \\
\hline C3:1-car & 216 & 85 & 100 & 20 & 1.00 \\
\hline C2-car & 204 & 85 & 100 & 20 & 1.45 \\
\hline C3-car & 218 & 85 & 100 & 20 & 2.86 \\
\hline C6:OH & 276 & 85 & 100 & 20 & 3.11 \\
\hline C5:OH-car & 262 & 85 & 120 & 20 & 3.45 \\
\hline C6:2-car & 256 & 85 & 100 & 20 & 4.00 \\
\hline C4-car & 232 & 85 & 100 & 20 & 4.38 \\
\hline C5:1-car & 244 & 85 & 100 & 20 & 5.09 \\
\hline C5-car & 246 & 85 & 100 & 20 & 5.44 \\
\hline C6:1-car & 258 & 85 & 100 & 20 & 5.74 \\
\hline C6-car & 260 & 85 & 120 & 20 & 6.57 \\
\hline $\mathrm{C} 8: \mathrm{OH}-\mathrm{car}$ & 304 & 85 & 100 & 20 & 6.60 \\
\hline C7-car & 274 & 85 & 100 & 20 & 7.13 \\
\hline C8:1-car & 286 & 85 & 100 & 20 & 7.44 \\
\hline C8-car & 288 & 85 & 120 & 20 & 8.22 \\
\hline C9-car & 302 & 85 & 100 & 20 & 8.53 \\
\hline C10:1-car & 314 & 85 & 100 & 20 & 9.03 \\
\hline C10-car & 316 & 85 & 120 & 20 & 9.55 \\
\hline C11-car & 330 & 85 & 100 & 20 & 9.89 \\
\hline C12:1-car & 342 & 85 & 100 & 20 & 10.14 \\
\hline C14:1:OH-car & 386 & 85 & 100 & 20 & 10.15 \\
\hline C14:OH-car & 388 & 85 & 100 & 20 & 10.61 \\
\hline C14:2-car & 368 & 85 & 100 & 20 & 10.65 \\
\hline C12-car & 344 & 85 & 120 & 20 & 10.97 \\
\hline C13-car & 358 & 85 & 100 & 20 & 11.01 \\
\hline C14:1-car & 370 & 85 & 100 & 20 & 11.15 \\
\hline C16:2-car & 396 & 85 & 100 & 20 & 11.38 \\
\hline C14-car & 372 & 85 & 140 & 20 & 11.61 \\
\hline C15-car & 386 & 85 & 100 & 20 & 11.86 \\
\hline C16:1-car & 398 & 85 & 100 & 20 & 11.90 \\
\hline
\end{tabular}


Table S2 Mass parameters, transitions and retention times for profiling acylcarnitines.

\begin{tabular}{llllll}
$\begin{array}{llll}\text { Compound } \\
\text { Name }\end{array}$ & $\begin{array}{l}\text { Precursor Ion } \\
(\mathrm{m} / \mathrm{z})\end{array}$ & $\begin{array}{l}\text { Product Ion } \\
(\mathrm{m} / \mathrm{z})\end{array}$ & $\begin{array}{l}\text { Fragmentor } \\
(\mathrm{V})\end{array}$ & $\begin{array}{l}\text { Collision Energy } \\
(\mathrm{V})\end{array}$ & $\begin{array}{l}\text { Rt } \\
(\mathrm{min})\end{array}$ \\
\hline C18:2-car & 424 & 85 & 100 & 20 & 12.13 \\
C18:3-car & 422 & 85 & 100 & 20 & 12.13 \\
C16-car & 400 & 85 & 140 & 20 & 12.43 \\
C18:1-car & 426 & 85 & 100 & 20 & 12.63 \\
C20:2-car & 452 & 85 & 160 & 20 & 12.79 \\
C17-car & 414 & 85 & 100 & 20 & 12.82 \\
C18-car & 428 & 85 & 160 & 20 & 13.19 \\
C20:1-car & 454 & 85 & 160 & 20 & 13.28 \\
C19-car & 442 & 85 & 100 & 20 & 13.60 \\
C20-car & 456 & 85 & 160 & 20 & 13.85 \\
\hline
\end{tabular}


Table S3 Peak area table of targeted metabolomics analysis in liver samples. Only the targeted metabolites with RSD no more than 20\% in QC samples are shown.

\begin{tabular}{|c|c|c|c|c|c|c|c|c|c|c|c|c|c|c|c|c|c|c|}
\hline Sample & S1 & S2 & S3 & S4 & S5 & S6 & S7 & S8 & S9 & S10 & S11 & S12 & S13 & S14 & S15 & S16 & S17 & S18 \\
\hline Treatment & Control & Control & Control & Control & Control & Control & Control & Control & Control & Control & VCZ & VCZ & VCZ & VCZ & VCZ & VCZ & VCZ & $\mathrm{VCZ}$ \\
\hline $17 \alpha$-Hydroxypreg & 79.33 & 53.53 & 33426.07 & 80.76 & 01.25 & 35.27 & 809.9 & 52241.16 & 632.35 & 583.65 & 20211.82 & 30544.46 & 14949.73 & 21617.03 & 29486.85 & 33185.08 & 2034.71 & 9950.47 \\
\hline 1-Methylhistidine & 5414.2 & 38188.88 & 48508.93 & 37348.56 & 55042.23 & 49925.78 & 42639.55 & 48517.65 & 33258.15 & 65153.99 & 126069.6 & 32528.7 & 50096.25 & 57631.76 & 30626.99 & 198773.4 & 51893.58 & 70826.05 \\
\hline 3-Methylh & 6068.091 & 7223.245 & 8980.203 & 6945.394 & 429.95 & 8742.902 & 6978.467 & 8079.453 & 6529.074 & 11239.29 & 15551.33 & 6001.517 & 10664.88 & 11314.55 & 6375.676 & 26392.4 & 9967.983 & 15319.96 \\
\hline Acetylcarnitine & 26278.27 & 34144.83 & 48870.75 & 34097.6 & 39174.88 & 39897.24 & 44467.77 & 33983.91 & 24680.78 & 40810.21 & 1466.526 & 16877.56 & 3125.775 & 2619.414 & 26477.8 & 3059.37 & 18083.53 & 3031.118 \\
\hline Alanine & 9027273 & 8946235 & 10311926 & 13410918 & 20559071 & 13401566 & 10099585 & 8450962 & 6693111 & 9907463 & 4748824 & 5927427 & 5946257 & 7779027 & 16538132 & 6134037 & 13204913 & 4684810 \\
\hline Asparagine & 3649 & 283066.3 & 312933.1 & 167220.5 & 261753.4 & 145786.2 & 178095.1 & 183015.5 & 200757.7 & 330787.4 & 43936.78 & 126476.7 & 124616 & 47868.99 & 212445.8 & 100813.2 & 146210 & 67298.16 \\
\hline Aspartate & 252267.7 & 211212.9 & 343018.7 & 388579.9 & 390087.6 & 233553.2 & 329277.5 & 296099.1 & 267784.9 & 259795.9 & 92935.22 & 286236.1 & 385852.3 & 213982.8 & 348922.1 & 181833 & 508444.8 & 268083.7 \\
\hline$\beta$-alanine & 195421.9 & 229203.7 & 294825.5 & 272638.9 & 333507.4 & 450301.6 & 301681.7 & 239635 & 374007.8 & 373079.9 & 176660.9 & 159412.7 & 291470.2 & 325496 & 331317.5 & 407619.8 & 368723 & 304661.2 \\
\hline Betaine & 3822.9 & 104579.6 & 108482.2 & 62831.78 & 93587.18 & 89941.04 & 78499.56 & 82082.54 & 86092.58 & 85424.57 & 34308.12 & 43087.76 & 55461.79 & 56190.13 & 62490.66 & 105249.8 & 28774.97 & 48338.87 \\
\hline$\beta$-N-Acetyl & 30571.56 & 27201.46 & 43798.43 & 36462.81 & 22471.9 & 53539.58 & 44984 & 52381.77 & 29428.45 & 52030.9 & 30325.45 & 43603.55 & 41246.99 & 56820.89 & 45004.05 & 42250.28 & 30481.67 & 48869.29 \\
\hline Billirubin & 2159.854 & 2254.197 & 7166.595 & 3253.194 & 3118.843 & 3120.072 & 2724.958 & 3265.653 & 5351.871 & 7443.895 & 5933.375 & 2661.741 & 5934.112 & 3258.478 & 1652.387 & 3820.061 & 1654.53 & 1790.844 \\
\hline Cheno & 87.06 & 947.3866 & 1167.144 & 1064.516 & 1268.835 & 888.5844 & 19.99 & 987.2152 & 860.1226 & 1349.136 & 1095.653 & 1036.094 & 1062.339 & 9.722 & 1099.475 & 920.493 & 962.4568 & 1246.89 \\
\hline Cholate & 2270.153 & 1423.26 & 13458.57 & 1577.117 & 5387.41 & 1369.83 & 2853.286 & 3156.936 & 2212.701 & 83544.02 & 8767.187 & 1242.723 & 4746.928 & 375.2198 & 582.5441 & 1998.221 & 554.9054 & 1299.898 \\
\hline Citrulline & 117909.3 & 199616.3 & 230767.5 & 107304.3 & 224486.1 & 275106 & 116832.1 & 108692.6 & 146086.1 & 274540.8 & 652277 & 62527.88 & 152565.5 & 348594.5 & 263472.8 & 655135.8 & 401990.5 & 226560.1 \\
\hline Corticosterone & 793.5851 & 914.9563 & 848.9538 & 839.176 & 609.2928 & 933.8195 & 931.5349 & 858.9109 & 900.4034 & 1365.724 & 686.4063 & 651.2927 & 869.4903 & 971.6232 & 695.5239 & 959.541 & 662.1707 & 857.2307 \\
\hline Creatine & 609421.7 & 936846.3 & 1390465 & 1008685 & 1147222 & 1318617 & 1284730 & 1394233 & 1173453 & 1459235 & 2818407 & 620356.7 & 949863.6 & 1163541 & 1062937 & 1669110 & 1058669 & 1739682 \\
\hline Creatinine & 10632.06 & 13161.28 & 15924.18 & 11263.77 & 16557.55 & 14300.9 & 14207.31 & 12063.99 & 15819.71 & 18648.5 & 13992.67 & 10182.7 & 11371.8 & 16124.36 & 21487.74 & 19898.81 & 19575.73 & 14281.68 \\
\hline
\end{tabular}




\begin{tabular}{|c|c|c|c|c|c|c|c|c|c|c|c|c|c|c|c|c|c|c|}
\hline Cytidine & 112643 & 89909.89 & 69665.85 & 123086.6 & 97911.26 & 82514.51 & 113056.3 & 171084.2 & 332335.2 & 276942.3 & 696793.3 & 198739.2 & 210159.1 & 306725.4 & 125495.6 & 260804.6 & 266625.8 & 288351.8 \\
\hline Dimethyl Glycine & 559504.7 & 434660.8 & 487856.3 & 405157.9 & 565768.4 & 696275.3 & 351908.3 & 491285.2 & 849988.9 & 506355.5 & 298038.1 & 204421.2 & 381481.3 & 302665 & 724246.4 & 650085 & 417230.2 & 275867.3 \\
\hline Fumarate & 71599.62 & 50106.58 & 73955.11 & 132823.6 & 70083.25 & 36717.62 & 79702.36 & 87104.65 & 69811.31 & 101953.4 & 188738.5 & 77865.33 & 91789.32 & 110244 & 114136.5 & 75735.69 & 129022.3 & 102509.9 \\
\hline GABA & 24409.79 & 21028.74 & 33387.12 & 23472.4 & 25013.15 & 26708.79 & 34143.82 & 32964.64 & 52888.03 & 48124.31 & 17610.22 & 22621.31 & 14249.43 & 13657.07 & 23796.38 & 18348.88 & 18122.98 & 27338.2 \\
\hline Glutamic acid & 69552.03 & 87126.71 & 203569.7 & 100284.2 & 200756.8 & 62929.5 & 77891.42 & 103716.5 & 89595.38 & 198055.1 & 643184.2 & 415957 & 604233.7 & 647281.4 & 256958 & 895045 & 1011144 & 1334236 \\
\hline Glutamine & 1627698 & 1648663 & 1708791 & 1207839 & 1457485 & 1180989 & 1528498 & 1206270 & 617909.2 & 1658582 & 442913.7 & 1941009 & 998184.2 & 1075307 & 1560049 & 1003879 & 916431.2 & 1538187 \\
\hline Glycine & 1033094 & 1475315 & 1327250 & 946060.8 & 1468060 & 1435052 & 1215417 & 1379126 & 1258401 & 1900611 & 1612275 & 1405045 & 2012451 & 2261588 & 986703.5 & 1837841 & 1783924 & 1988432 \\
\hline Glycocholate & 243.9782 & 148.3151 & 4084.972 & 268.3138 & 221.5723 & 58.14468 & 558.9127 & 651.4868 & 530.3789 & 19494.58 & 748.0526 & 471.8786 & 652.4938 & 221.0631 & 452.9419 & 50.26925 & 339.5763 & 290.7847 \\
\hline Hippuric acid & 1245.982 & 5936.855 & 4654.74 & 5202.846 & 3296.126 & 8717.987 & 2700.923 & 1687.043 & 2258.862 & 13357.8 & 1808.992 & 4829.67 & 2072.312 & 5203.991 & 5781.05 & 3463.602 & 3362.725 & 2204.19 \\
\hline Histidine & 1503261 & 2140184 & 2112779 & 1291774 & 1697300 & 1672295 & 1101509 & 1004727 & 1143229 & 2373054 & 1197470 & 1617982 & 1913653 & 2026348 & 2609455 & 2387976 & 2365511 & 2298234 \\
\hline Inosine & 14393518 & 24701434 & 7238213 & 26562658 & 25515482 & 25459299 & 21314919 & 25772828 & 24337371 & 11720698 & 13600790 & 13617514 & 7463858 & 12251071 & 16210151 & 10839168 & 16096991 & 10625365 \\
\hline Isoleucine & 2788194 & 4021146 & 4431335 & 2983062 & 3705514 & 3942132 & 3545684 & 3610223 & 3653358 & 4093611 & 1604362 & 2184928 & 2382590 & 2878733 & 3061629 & 3475285 & 1569085 & 2265312 \\
\hline Lysine & 1117383 & 1500604 & 1633366 & 1011910 & 1398329 & 1765224 & 1152092 & 1208677 & 1466079 & 1443080 & 609527 & 964471.1 & 987399.6 & 1190937 & 1278331 & 1201466 & 1444926 & 903685 \\
\hline Methionine & 78409.65 & 169140.5 & 227925.8 & 157475.6 & 153364.1 & 228517.8 & 179974 & 210270.7 & 268057.9 & 263271.7 & 76843.13 & 111347.9 & 87734.02 & 98046.55 & 158732.5 & 98785.45 & 98811.89 & 72210.39 \\
\hline Ornithine & 357594.3 & 572327.4 & 610855.6 & 515953.9 & 521511.1 & 592561 & 515896.6 & 558568.1 & 767429.1 & 641120.5 & 444870.8 & 274956.5 & 405168.6 & 352870.5 & 560574.2 & 403403.1 & 498308.8 & 386580 \\
\hline Phenylalanine & 668712.6 & 1037284 & 1215766 & 909333.8 & 1135947 & 1301932 & 1283861 & 1073682 & 1333133 & 1289012 & 317705.6 & 537736.2 & 494929.7 & 443754.6 & 839477.1 & 527780.3 & 413584.1 & 398717.4 \\
\hline Pyroglutamate & 161157.2 & 188163.6 & 202872.5 & 256562.1 & 307134 & 190883.8 & 159867.7 & 171984.5 & 158976.1 & 185978.4 & 406283.2 & 410168.6 & 589630.9 & 428477.6 & 222433.2 & 641902.5 & 305957.7 & 389555 \\
\hline Pyruvate & 4332.808 & 34276.97 & 25515.83 & 5894.783 & 4430.978 & 7958.101 & 2716.305 & 5314.362 & 63908.39 & 44786.43 & 7043.666 & 16095.57 & 60673.43 & 94778.56 & 4844.443 & 9645.168 & 11344.76 & 10361.55 \\
\hline Serine & 401182.2 & 498713.2 & 606488.7 & 386820.5 & 499110 & 700700.9 & 567947.9 & 571800.5 & 574947.9 & 730303 & 225419.2 & 387154.8 & 291318.1 & 387866 & 380418.1 & 315739.9 & 372296.2 & 297365.2 \\
\hline Taurine & 1634853 & 1275603 & 1404024 & 1456629 & 1131000 & 1466342 & 1489458 & 1010812 & 2038178 & 1018694 & 1418649 & 1569321 & 1306143 & 1650225 & 1799179 & 1385486 & 1499979 & 1316845 \\
\hline Threonine & 419738.4 & 630271.7 & 706766.4 & 396579.1 & 605059.2 & 670172.4 & 576630.4 & 459518 & 487646.7 & 811403.7 & 383366.8 & 265616.7 & 584088.2 & 694694.4 & 508394.6 & 734656.3 & 320228.2 & 403620.7 \\
\hline
\end{tabular}




\begin{tabular}{|c|c|c|c|c|c|c|c|c|c|c|c|c|c|c|c|c|c|c|}
\hline TMAO & 11713.95 & 3030.079 & 5449.627 & 13766.9 & 5821.431 & 4102.962 & 8782.23 & 6062.728 & 7928.498 & 9481.223 & 19611.9 & 8240.514 & 14710 & 9427.011 & 10477.7 & 20074.87 & 16601.94 & 18224.29 \\
\hline Tryptophan & 10559.1 & 418215.7 & 528032.9 & 386841.2 & 500815.4 & 441449.4 & 534379 & 457909.5 & 504914.5 & 474179.5 & 283639.7 & 322861 & 291159.8 & 306274.9 & 460377 & 292394.8 & 216033.7 & 273696.3 \\
\hline Tyrosine & 364130.5 & 574336.1 & 717738.3 & 531512.3 & 542568.9 & 753635.3 & 608847.3 & 615528.8 & 631584.6 & 686126.1 & 216599.1 & 288642.8 & 345705.7 & 352056.2 & 485780.5 & 373577 & 285694.6 & 205700.8 \\
\hline Urea & 211020 & 3782770 & 4440235 & 4206936 & 3938590 & 5086394 & 3047170 & 3437748 & 2897486 & 3369283 & 6200739 & 2326685 & 6699029 & 4625856 & 3004199 & 9785182 & 5535596 & 6289545 \\
\hline Ureido & 758.42 & 148494.8 & 227942.2 & 151688.2 & 154765.2 & 197971.3 & 187490.7 & 189950.9 & 167709.4 & 249774.9 & 419076.4 & 113530.3 & 145602.1 & 177651.8 & 169048.2 & 267187.6 & 211150.7 & 270895 \\
\hline Uridine & 075.321 & 3262.198 & 2962.711 & 4632.377 & 2470.708 & 3727.268 & 3737.853 & 7749.85 & 10501.99 & 13700.84 & 28092.86 & 7786.457 & 8645.398 & 11440.7 & 5591.491 & 9832.523 & 10841.99 & 12751.35 \\
\hline Urocanate & 060.316 & 5327.313 & 3871.251 & 3015.028 & 5556.254 & 4109.425 & 5678.306 & 8510.569 & 5431.369 & 5040.889 & 8167.399 & 4502.171 & 2536.484 & 2493.582 & 4571.235 & 4506.304 & 3389.349 & 3648.132 \\
\hline Ursodeoxycholic acid & 1676.734 & 2502.072 & 1787.337 & 1721.194 & 1560.358 & 1789.386 & 1627.118 & 1993.57 & 1129.027 & 1655.109 & 963.6433 & 1670.313 & 1911.303 & 1662.854 & 1381.152 & 1600.725 & 1446.844 & 1258.423 \\
\hline Valine & 1199553 & 1855491 & 1898343 & 1149737 & 1368800 & 1663689 & 1383958 & 1516684 & 420966 & 1548167 & 600079.2 & 792631.2 & 916949.1 & 1077014 & 1087502 & 1984641 & 544774.7 & 772613.4 \\
\hline
\end{tabular}


Table S4 Peak area table of targeted metabolomics analysis in plasma samples. Only the targeted metabolites with RSD no more than $20 \%$ in QC samples are shown.

\begin{tabular}{|c|c|c|c|c|c|c|c|c|c|c|c|c|c|c|c|c|c|c|}
\hline Sample & 1 & 2 & 3 & 4 & 5 & 6 & 7 & 8 & 9 & 10 & 11 & 12 & 13 & 14 & 15 & 16 & 17 & 18 \\
\hline Treatment & ontrol & Control & Control & Control & Control & Control & Control & Control & ontrol & Control & VCZ & VCZ & VCZ & VCZ & vCZ & $\mathrm{vCZ}$ & $\mathrm{VCZ}$ & $\mathrm{vCZ}$ \\
\hline $17 \alpha$-Hydroxypregnenolone & 65033.32 & 132848.7 & 141039.2 & 186682.7 & 176861.8 & 223555.2 & 180323.4 & 180475.8 & 172112.7 & 79417.23 & 96059.8 & 31787.33 & 37714.27 & 33509.63 & 138243.9 & 88451.76 & 153585.7 & 101186.7 \\
\hline 1-Methylhistidine & 940958 & 1084766 & 1093655 & 861329.4 & 1095595 & 928650.8 & 921974.7 & 852799.8 & 694105.6 & 1191107 & 1139273 & 696332.7 & 962143.6 & 1076241 & 682195.4 & 2485582 & 1339761 & 1676116 \\
\hline 3-Methylhistidine & 3591.1 & 127786.7 & 137585.7 & 108237.4 & 119610 & 102440.7 & 103967.6 & 108040.1 & 81423.29 & 147298.6 & 129034.1 & 89731.24 & 109918.1 & 120019 & 81133.68 & 289827.9 & 161369.1 & 172894 \\
\hline 5-Hydroxylysine & 11852.13 & 16039.23 & 15772.77 & 13042.95 & 13393.55 & 11754.66 & 12163.08 & 14102.01 & 10916.86 & 10849.88 & 9300.689 & 14699.71 & 13233.05 & 12838.09 & 16750.28 & 14567.23 & 18401.78 & 10964.09 \\
\hline Acetoacetate & 70601.1 & 45400.37 & 10482.14 & 59520.78 & 50604.59 & 15372.18 & 4426.866 & 9950.389 & 22278.18 & 9823.016 & 55631.45 & 4678.881 & 70304.49 & 20633.89 & 6507.126 & 64785.95 & 46624.21 & 62132.64 \\
\hline Acetylcarnitine & 105814.1 & 87279.78 & 88857.01 & 97936.83 & 103358.2 & 93794.93 & 104090.4 & 115200.1 & 105950.8 & 107921.5 & 24889.7 & 61661.14 & 45670.45 & 37500.65 & 82822.59 & 28439.5 & 53687.61 & 30860.39 \\
\hline Alanine & 14415088 & 11402625 & 15604982 & 13506081 & 15289748 & 19455877 & 13848359 & 15443109 & 13739453 & 21164980 & 4696723 & 10952821 & 10139645 & 13155424 & 25686808 & 8380653 & 19355166 & 7648274 \\
\hline$\alpha$-Ketoglutarate & 8954.776 & 6953.535 & 10377.42 & 15180.76 & 16254.85 & 16783.42 & 21857.02 & 25798.68 & 38568.05 & 13287.55 & 17851.08 & 4420.461 & 4946.462 & 2071.708 & 23441.33 & 12439.29 & 6994.234 & 4099.839 \\
\hline Arginine & 94241.55 & 106095.9 & 104752.7 & 163668.7 & 133660.4 & 112252.2 & 102774.4 & 143005.5 & 137538.2 & 119585.5 & 65002.83 & 330654 & 246441.1 & 233377.5 & 234071.1 & 170171 & 219596.5 & 224444.6 \\
\hline Asparagine & 1314074 & 1033604 & 1076921 & 1287553 & 1158372 & 1317729 & 1008234 & 1119380 & 1288915 & 1207401 & 862816.6 & 1226400 & 1135029 & 1565659 & 1667861 & 1642153 & 2047867 & 1607182 \\
\hline Aspartate & 126288.5 & 77507.44 & 117941.4 & 108072.8 & 110491.3 & 85847.06 & 115362.3 & 98831.13 & 63553.43 & 60506.32 & 50554.25 & 111681.6 & 98678.63 & 126774.3 & 202486.3 & 116691.4 & 113076.5 & 70965.18 \\
\hline$\beta$-alanine & 19525.54 & 27957.04 & 23978.91 & 16445.53 & 15303.09 & 27459.31 & 21720.97 & 16954.33 & 37892.43 & 15676.07 & 10931.01 & 8122.318 & 12814.38 & 22284.09 & 31640.33 & 21396.9 & 13436.5 & 12014.65 \\
\hline Betaine & 562642.1 & 342679.1 & 505910.2 & 351591.9 & 404973.2 & 572325.4 & 436658.5 & 429699.8 & 467473.5 & 686541.9 & 313034 & 333555.8 & 686578.8 & 1111531 & 989919.2 & 1005056 & 652915.3 & 855336.3 \\
\hline$\beta$-N-Acetylglucosamine & 19150.68 & 32354.51 & 29531.29 & 23235.41 & 22176.04 & 26658.67 & 19812.71 & 16707.32 & 17279.44 & 14126.72 & 29147.99 & 15684.45 & 28475.71 & 25263.56 & 22356.34 & 26318.92 & 37527.53 & 22157.99 \\
\hline Cadaverine & 400448.8 & 342640.8 & 356448.7 & 356716.3 & 415062.5 & 391192.2 & 443244.9 & 473759.3 & 442745 & 349454.3 & 286920.7 & 325921.7 & 361090 & 365003.5 & 476144 & 345031.4 & 385710 & 322424.1 \\
\hline Chenodeoxycholic Acid & 1700.621 & 2513.847 & 2734.054 & 3987.796 & 3701.14 & 3577.892 & 4060.038 & 3292.965 & 4841.778 & 1617.226 & 3784.256 & .655 & 1483.123 & 1358.356 & 2953.493 & 4730.557 & 4982.326 & 2566.734 \\
\hline Cholate & 11596.47 & 14097.17 & 5853.003 & 29304.24 & 32999.53 & 14368.6 & 19557.37 & 6160.347 & 228156.5 & 34836.16 & 125429 & 3678.988 & 16390.84 & 7350.957 & 4513.082 & 24990.45 & 7637.148 & 8322.674 \\
\hline
\end{tabular}




\begin{tabular}{|c|c|c|c|c|c|c|c|c|c|c|c|c|c|c|c|c|c|c|}
\hline Citrulline & 1789603 & 1892068 & 1765530 & 1991097 & 1642438 & 1255842 & 1326435 & 1387182 & 1129835 & 2139402 & 6051515 & 1784474 & 2143321 & 2535278 & 2102048 & 5126018 & 3201022 & 4053820 \\
\hline Corticosterone & 1329.182 & 3445.933 & 3388.319 & 4019.047 & 3421.803 & 3680.264 & 3437.954 & 3393.474 & 3631.798 & 2212.81 & 7806.618 & 3229.571 & 2520.755 & 2619.289 & 4519.573 & 3052.313 & 7217.179 & 3377.832 \\
\hline Creatine & 8648774 & 8155446 & 7626222 & 10322064 & 8369810 & 8780456 & 8210995 & 7483255 & 7424432 & 10311073 & 7065667 & 8290723 & 11367777 & 12626899 & 13932110 & 9931651 & 7445849 & 13284330 \\
\hline Creatinine & 681764.7 & 745701.1 & 831782.8 & 907616.2 & 793333.5 & 931387.7 & 675987.4 & 590948.8 & 697845.4 & 949610.3 & 968309.4 & 654525 & 627493.6 & 838141.4 & 1077977 & 1050851 & 879222.5 & 726333.7 \\
\hline Cystine & 100568.5 & 338742.6 & 106668 & 91804.44 & 107097.1 & 82333.68 & 55100.45 & 61627.41 & 28863.98 & 62794.53 & 82963.03 & 466539.7 & 103580.4 & 173590.3 & 204308.8 & 292225.5 & 396004.7 & 156677.7 \\
\hline Cytidine & 1656345 & 1062417 & 1032822 & 1329852 & 1229775 & 1477353 & 1080259 & 1728355 & 2478484 & 2024987 & 883676.8 & 527086.8 & 1212549 & 1223312 & 1829326 & 1319340 & 486272.1 & 804967.7 \\
\hline Dimethyl Glycine & 2630823 & 3097353 & 3213673 & 3161396 & 3409695 & 3335659 & 3314905 & 3450337 & 4119217 & 2593324 & 1705489 & 1515618 & 2173066 & 2157402 & 2248588 & 4144109 & 2098082 & 1846587 \\
\hline Fumarate & 33964.06 & 20985.31 & 24577.5 & 43501.38 & 58245.96 & 46013.93 & 37786.96 & 53383.6 & 86996.7 & 36963.91 & 31508.44 & 19221.69 & 31260.61 & 18577 & 75969.76 & 32883.46 & 27153.17 & 19249.93 \\
\hline GABA & 3537.035 & 3630.148 & 3945.31 & 2522.082 & 2423.388 & 3111.335 & 3878.306 & 3166.793 & 38178.39 & 4237.616 & 11200.26 & 3032.226 & 7355.85 & 3908.209 & 5951.106 & 7462.465 & 4249.629 & 4298.644 \\
\hline Glutamic acid & 582825.2 & 576215 & 620343.1 & 943091.8 & 805984.4 & 887694.1 & 1152432 & 767987.2 & 960280.4 & 277027.8 & 802166.9 & 925380.6 & 1288051 & 1845139 & 2293733 & 1536635 & 588741.5 & 788213.3 \\
\hline Glutamine & 4183917 & 4010974 & 4603829 & 3155465 & 4026888 & 4093084 & 3539866 & 3536850 & 3413823 & 3911793 & 2652466 & 5796332 & 4187530 & 6150730 & 7426973 & 5208798 & 4963487 & 5656340 \\
\hline Glutathione & 9943.663 & 11413.17 & 7832.777 & 9566.594 & 8465.049 & 8661.203 & 8660.499 & 5589.279 & 2513.505 & 4962.829 & 1581.111 & 7883.141 & 4909.537 & 5746.237 & 15066.21 & 7958.323 & 11359.02 & 9265.621 \\
\hline Glycine & 3159368 & 3086376 & 3149337 & 2367161 & 2541391 & 3196868 & 2852218 & 2335538 & 3890171 & 3519226 & 1312870 & 1879302 & 1721572 & 2492292 & 1837332 & 1606934 & 2247825 & 1295997 \\
\hline Glycocholate & 207.4882 & 60.01456 & 59.70781 & 398.6601 & 247.5564 & 408.9461 & 179.1205 & 97.13877 & 4158.055 & 3091.195 & 829.7727 & 345.1611 & 257.5831 & 93.7951 & 350.0764 & 546.2068 & 356.2263 & 183.0144 \\
\hline Hippuric acid & 30720.76 & 33481.86 & 24384.83 & 53867.25 & 29867.35 & 107224.2 & 37093.93 & 32026.63 & 48110.68 & 169272.2 & 4757.668 & 8401.174 & 7681.461 & 9999.117 & 32419.28 & 9883.724 & 36111.69 & 4516.175 \\
\hline Histidine & 2906568 & 2793778 & 2942263 & 2441019 & 2613910 & 2711572 & 1868152 & 2116996 & 2850665 & 3635695 & 2382786 & 3016995 & 3856038 & 4856884 & 4801172 & 5146304 & 3484009 & 4460491 \\
\hline Inosine & 7127215 & 1849037 & 3159813 & 5081688 & 591391.4 & 4201627 & 5602188 & 4257415 & 1069313 & 1557309 & 4048.424 & 7191.339 & 4407.356 & 2329.205 & 36250.87 & 8081.47 & 1634.871 & 2717.608 \\
\hline Isoleucine & 14569253 & 7798827 & 12433087 & 9094574 & 8222137 & 13224004 & 8975688 & 8826101 & 10954482 & 20285287 & 7978246 & 7380360 & 18894879 & 33786503 & 24374622 & 25018611 & 12047934 & 27505854 \\
\hline Lithocholic acid & 17223.94 & 20594.06 & 20681.06 & 25784.51 & 20176.55 & 18914.43 & 18092.78 & 18356.44 & 24254.29 & 14570.48 & 30370.97 & 13080.22 & 18855.96 & 13896.37 & 15990.03 & 17223.41 & 12740.66 & 14016.43 \\
\hline Lysine & 30387894 & 25880136 & 28301849 & 28760002 & 23452741 & 24617029 & 25530856 & 22235726 & 20901586 & 21112844 & 12911361 & 28323239 & 31273605 & 47184690 & 47350120 & 29638322 & 45853032 & 29961495 \\
\hline Methionine & 542217.8 & 350651.9 & 549004.3 & 579574.7 & 492474.6 & 915599.6 & 805251.5 & 678814.3 & 1072821 & 1511614 & 375140.2 & 639523.4 & 697897.6 & 1500745 & 2852241 & 753089.2 & 1085287 & 829478.8 \\
\hline
\end{tabular}




\begin{tabular}{|c|c|c|c|c|c|c|c|c|c|c|c|c|c|c|c|c|c|c|}
\hline Ornithine & 2534128 & 2743448 & 1930674 & 2325535 & 2055653 & 1947456 & 2064096 & 2070039 & 2678803 & 3247671 & 2615793 & 3639664 & 4402670 & 5085326 & 5153957 & 3525933 & 5458632 & 3426795 \\
\hline Phenylalanine & 4808808 & 2189295 & 4503616 & 2930780 & 3311972 & 5924881 & 4276291 & 4485287 & 7569360 & 8318788 & 5442821 & 3299956 & 7222103 & 12352507 & 10681862 & 8422779 & 5848572 & 7546881 \\
\hline Pyroglutamate & 248431.2 & 94022.15 & 95983.52 & 383258.4 & 476871.6 & 261495.8 & 64352.04 & 54584.17 & 198737.6 & 262822.4 & 221598.2 & 498604.4 & 175354.3 & 116459.2 & 344836.5 & 481612.2 & 141253.6 & 400834.5 \\
\hline Pyruvate & 429764.3 & 240522.8 & 108287 & 15579.48 & 72760.73 & 24907.72 & 266499.5 & 28118.96 & 108866.9 & 58834.62 & 65910.75 & 14505.22 & 369704.2 & 70494.82 & 30603.17 & 87401.76 & 198131.7 & 69206.72 \\
\hline Serine & 1850425 & 1716307 & 1684921 & 1565279 & 1552659 & 1835230 & 1629593 & 1399702 & 1362827 & 1764743 & 595361 & 1865263 & 1418543 & 1857885 & 2385437 & 1419075 & 2511742 & 1378282 \\
\hline Taurine & 137997 & 8211.803 & 4633.399 & 161428.3 & 147279.9 & 170679.5 & 4472.441 & 5880.351 & 394089.4 & 81282.85 & 200401.8 & 105852.4 & 37310.99 & 147923.2 & 250413.8 & 299273.6 & 1161.37 & 124882.9 \\
\hline Threonine & 1439297 & 2271510 & 1694260 & 1674072 & 1634687 & 2227450 & 1714994 & 1533530 & 2138806 & 2508990 & 1285436 & 2062520 & 2577872 & 4233643 & 3392963 & 2589016 & 3575615 & 2761816 \\
\hline TMAO & 10061.33 & 4949.586 & 2223.875 & 3062.525 & 1621.847 & 338.0763 & 1464.915 & 1292.221 & 4051.641 & 2924.248 & 151667 & 31410.06 & 97453.66 & 75724.23 & 1400.37 & 10729.91 & 2530.59 & 802.5238 \\
\hline Tryptophan & 2425439 & 1513322 & 1972851 & 1832485 & 2244016 & 2036885 & 2414545 & 2475183 & 3119434 & 2647311 & 1015771 & 2414143 & 2682866 & 3535610 & 2938940 & 1651655 & 1524630 & 1695783 \\
\hline Tyrosine & 1183346 & 1063883 & 1464075 & 1196437 & 1278596 & 1754198 & 1226308 & 1560245 & 1807893 & 1411909 & 1342961 & 677028.2 & 1378484 & 1859776 & 2250130 & 1417992 & 1286391 & 1219232 \\
\hline Uridine diphosphate (UDP) & 1859.403 & 2667.426 & 2293.435 & 3011.377 & 2480.402 & 2665.748 & 2762.053 & 2933.971 & 2748.235 & 1711.862 & 7498.687 & 2355.711 & 1861.851 & 1729.403 & 2759.931 & 2697.562 & 4448.962 & 4822.25 \\
\hline Urea & 17762684 & 11579135 & 11141899 & 20502305 & 18243727 & 20578867 & 11883146 & 11807462 & 15049531 & 23320105 & 33968290 & 11251803 & 21894248 & 21784215 & 22633127 & 35911297 & 13930585 & 26623839 \\
\hline Ureidopropionic acid & 1975824 & 1827641 & 1471245 & 2330701 & 1793126 & 1731917 & 1691919 & 1647231 & 1530230 & 2109310 & 1628891 & 1479560 & 2443979 & 2527279 & 2865357 & 2558772 & 2311138 & 2649454 \\
\hline Uridine & 62698.4 & 40467.86 & 42567.33 & 55582.6 & 49043.49 & 58784.55 & 45338 & 58094.3 & 90692.81 & 82246.27 & 32154.25 & 19548.57 & 48583.57 & 46819.65 & 59991.31 & 55040.94 & 20035.87 & 31030.17 \\
\hline Urocanate & 27856.57 & 22502.01 & 32370.47 & 34572.08 & 35961.15 & 26523.85 & 22643.7 & 33133.34 & 42880.9 & 34920.17 & 43935.04 & 30898.39 & 34054.68 & 30255.99 & 25440.86 & 43120.98 & 41396.14 & 28016.17 \\
\hline Valine & 8078535 & 5719336 & 7657875 & 4762854 & 5926295 & 8467153 & 5875051 & 6323672 & 6407890 & 11206234 & 4430530 & 4958625 & 10517211 & 16527789 & 13958188 & 13668652 & 9526171 & 12025687 \\
\hline
\end{tabular}


Table S5 Metabolites with fold change less than 0.8 or over 1.2 in liver and plasma samples.

\begin{tabular}{|c|c|c|c|c|c|c|}
\hline & \multicolumn{3}{|c|}{ Liver } & \multicolumn{3}{|c|}{ Plasma } \\
\hline & $\begin{array}{c}\text { Fold } \\
\text { Change }\end{array}$ & $p$-value & FDR & $\begin{array}{c}\text { Fold } \\
\text { Change }\end{array}$ & $p$-value & FDR \\
\hline \multicolumn{7}{|l|}{ Amino acids } \\
\hline 1-Methylhistidine & 1.79 & & & 1.34 & & \\
\hline 3-Methylhistidine & 1.63 & $3.62 \mathrm{E}-02$ & 7.39E-02 & 1.31 & & \\
\hline Alanine & 0.72 & & & NA & & \\
\hline Arginine & NA & & & 1.77 & $1.13 \mathrm{E}-02$ & $5.56 \mathrm{E}-02$ \\
\hline Asparagine & 0.53 & $5.12 \mathrm{E}-03$ & $1.41 \mathrm{E}-02$ & 1.25 & & \\
\hline$\beta$-alanine & NA & & & 0.72 & & \\
\hline Cystine & NA & & & 2.17 & $1.71 \mathrm{E}-02$ & 7.10E-02 \\
\hline Dimethyl Glycine & 0.76 & & & 0.68 & $1.24 \mathrm{E}-03$ & 8.37E-03 \\
\hline Glycine & 1.35 & $1.41 \mathrm{E}-02$ & $3.31 \mathrm{E}-02$ & 0.61 & $1.00 \mathrm{E}-04$ & $1.35 \mathrm{E}-03$ \\
\hline Glutamic acid & 6.56 & 6.85 E-07 & $3.22 \mathrm{E}-05$ & 1.55 & & \\
\hline Glutamine & NA & & & 1.37 & 2.27 E-02 & $8.05 \mathrm{E}-02$ \\
\hline Hippuric acid & NA & & & 0.32 & 5.77 E-04 & $6.23 \mathrm{E}-03$ \\
\hline Histidine & 1.35 & $3.15 \mathrm{E}-02$ & $6.73 \mathrm{E}-02$ & 1.55 & $1.23 \mathrm{E}-03$ & 8.37E-03 \\
\hline Phenylalanine & 0.45 & 7.35 E-06 & 1.73 E-04 & 1.71 & $1.45 \mathrm{E}-02$ & $6.54 \mathrm{E}-02$ \\
\hline Pyroglutamate & 2.12 & $9.18 \mathrm{E}-05$ & $1.11 \mathrm{E}-03$ & 1.43 & & \\
\hline Isoleucine & 0.67 & $1.26 \mathrm{E}-03$ & $6.12 \mathrm{E}-03$ & 1.88 & $2.54 \mathrm{E}-02$ & 8.05E-02 \\
\hline Lysine & 0.79 & 3.87 E-02 & 7.58 E-02 & 1.33 & & \\
\hline Methionine & 0.54 & $1.30 \mathrm{E}-03$ & $6.12 \mathrm{E}-03$ & 1.73 & & \\
\hline Serine & 0.62 & $1.38 \mathrm{E}-04$ & 1.30 E-03 & NA & & \\
\hline Threonine & NA & & & 1.55 & $1.11 \mathrm{E}-02$ & $5.56 \mathrm{E}-02$ \\
\hline Tryptophan & 0.67 & $6.39 \mathrm{E}-04$ & 4.29 E-03 & NA & & \\
\hline Tyrosine & 0.54 & 9.46 E-05 & $1.11 \mathrm{E}-03$ & NA & & \\
\hline Urocanate & 0.82 & & & NA & & \\
\hline Valine & 0.65 & $4.50 \mathrm{E}-03$ & $1.32 \mathrm{E}-02$ & 1.63 & 3.22 E-02 & 8.05E-02 \\
\hline \multicolumn{7}{|l|}{ Nucleosides } \\
\hline Cytidine & 2.22 & 3.56 E-03 & $1.19 \mathrm{E}-02$ & 0.71 & & \\
\hline Inosine & 0.58 & $1.11 \mathrm{E}-02$ & 2.76 E-02 & 0.0023 & 3.00 E-10 & $1.62 \mathrm{E}-08$ \\
\hline Uridine & 2.48 & 1.17 E-03 & 6.12 E-03 & 0.70 & 3.02 E-02 & 8.05E-02 \\
\hline
\end{tabular}


Table S5 Metabolites with fold change less than 0.8 or over 1.2 in liver and plasma samples. (continued)

\begin{tabular}{|c|c|c|c|c|c|c|}
\hline & \multicolumn{3}{|c|}{ Liver } & \multicolumn{3}{|c|}{ Plasma } \\
\hline & $\begin{array}{c}\text { Fold } \\
\text { Change }\end{array}$ & $p$-value & FDR & $\begin{array}{c}\text { Fold } \\
\text { Change }\end{array}$ & $p$-value & FDR \\
\hline \multicolumn{7}{|l|}{ Urea cycle metabolites } \\
\hline Urea & 1.47 & & & 1.53 & 3.01 E-02 & $8.05 \mathrm{E}-02$ \\
\hline Ornithine & 0.75 & 1.09 E-02 & 2.76 E-02 & 1.84 & 2.22 E-05 & $4.00 \mathrm{E}-04$ \\
\hline Citrulline & 2.04 & & & 2.14 & 8.07 E-04 & $7.26 \mathrm{E}-03$ \\
\hline \multicolumn{7}{|l|}{ Energy metabolites } \\
\hline Pyruvate & 1.57 & & & 0.79 & & \\
\hline Fumarate & 1.49 & $2.03 \mathrm{E}-02$ & $4.55 \mathrm{E}-02$ & 0.71 & & \\
\hline Ureidopropionic acid & 1.32 & & & 1.30 & $1.87 \mathrm{E}-02$ & $7.20 \mathrm{E}-02$ \\
\hline UDP & NA & & & 1.35 & & \\
\hline $\begin{array}{c}\text { a-Ketoglutarate } \\
\text { (2-Oxoglutaric acid) }\end{array}$ & NA & & & 0.53 & $3.22 \mathrm{E}-02$ & $8.05 \mathrm{E}-02$ \\
\hline \multicolumn{7}{|l|}{ Bile acids } \\
\hline Cholate & 0.65 & & & 0.62 & & \\
\hline Glycocholate & 0.54 & & & 0.57 & & \\
\hline Lithocholic acid & NA & & & 0.83 & & \\
\hline \multicolumn{7}{|l|}{ Others } \\
\hline $\begin{array}{c}17 \alpha- \\
\text { Hydroxypregnenolone }\end{array}$ & 0.59 & $2.85 \mathrm{E}-03$ & $1.09 \mathrm{E}-02$ & 0.52 & $7.95 \mathrm{E}-03$ & 4.77E-02 \\
\hline Acetoacetate & NA & & & 1.29 & & \\
\hline Betaine & 0.63 & $2.87 \mathrm{E}-03$ & $1.09 \mathrm{E}-02$ & 1.64 & $3.14 \mathrm{E}-02$ & $8.05 \mathrm{E}-02$ \\
\hline Creatine & 1.21 & & & 1.26 & & \\
\hline Corticosterone & NA & & & 1.30 & & \\
\hline GABA & 0.64 & $3.99 \mathrm{E}-03$ & $1.25 \mathrm{E}-02$ & 0.83 & & \\
\hline Taurine & NA & & & 1.27 & & \\
\hline TMAO & 1.98 & 3.00 E-03 & 1.09 E-02 & 14.39 & $3.28 \mathrm{E}-02$ & $8.05 \mathrm{E}-02$ \\
\hline
\end{tabular}

${ }^{* 1}$ NA: fold change in the range of $0.8 \sim 1.2$.

$* 2$ Only $p$-value $<0.05$ were showed in table.

${ }^{* 3}$ One outlier in control group (no.10) was excluded. 